Tools and Techniques

\title{
Cre-loxP-based system for removal and reuse of selection markers in Ashbya gossypii targeted engineering
}

\author{
Tatiana Q. Aguiar ${ }^{1}$, Cláudia Dinis ${ }^{1}$, Lucília Domingues* \\ CEB - Centre of Biological Engineering, University of Minho, 4710-057 Braga, Portugal
}

\section{A R T I C L E I N F O}

Article history:

Received 8 August 2013

Accepted 21 April 2014

Available online 2 May 2014

\section{Keywords:}

Ashbya gossypii

Cre-loxP system

Gene targeting

Marker recycling

Recombinase expression

\begin{abstract}
A B S T R A C T
The filamentous ascomycete Ashbya gossypii is amenable to genetic manipulation and is an excellent model system for studying eukaryotic cell biology. However, the number of selection markers in current use for both targeted gene integration and disruption in this fungus are very limited. Therefore, the Cre-loxP recombination system was adapted for use in A. gossypii and its effectiveness in recycling marker genes was demonstrated by constructing both single and double deleted Agura3 and Agade1 auxotrophic strains free of exogenous markers. In spite of its wide use, this is the first report in which the Cre-loxP system was applied to A. gossypii, opening new perspectives for targeted engineering of this fungus with several promising biotechnological applications.
\end{abstract}

(c) 2014 Elsevier Inc. All rights reserved.

\section{Introduction}

The Cre-loxP recombination system of the bacteriophage P1 has been shown to mediate efficient recombination between directly repeated loxp sites flanking selectable markers in several organisms, resulting in the excision of marker genes (Sternberg and Hamilton, 1981; Sauer and Henderson, 1988; Orban et al., 1992; Zhang et al., 2009). Cre-loxP-based systems have been widely used to study gene function and to remove selection markers in fungi, among which Saccharomyces cerevisiae (Sauer, 1987; Güldener et al., 1996), Kluyveromyces lactis (Steensma and Ter Linde, 2001), Kluyveromyces marxianus (Ribeiro et al., 2007), Schizosaccharomyces pombe (Iwaki and Takegawa, 2004), Yarrowia lipolytica (Fickers et al., 2003), Hansenula polymorpha (Qian et al., 2009), Cryptococcus neoformans (Patel et al., 2010), Aspergillus nidulans (Forment et al., 2006), Trichoderma reesei (Steiger et al., 2011), Neotyphodium coenophialum, N. uncinatum and Epichloë festucae (Florea et al., 2009).

Ashbya gossypii (syn. Eremothecium gossypii) is a flavinogenic filamentous ascomycete (Ashby and Nowell, 1926; Kurtzman, 1995) used for the industrial production of riboflavin (Stahmann et al., 2000), which shares remarkable genomic similarities with the budding yeast S. cerevisiae (Brachat et al., 2003; Dietrich et al., 2004), being therefore considered an excellent model to study filamentous growth (Wendland and Walther, 2005; Schmitz and

\footnotetext{
* Corresponding author. Fax: +351253604429.

E-mail address: luciliad@deb.uminho.pt (L. Domingues).

1 These authors contributed equally to this work.
}

Philippsen, 2011). The high degree of gene homology and gene order conservation (synteny) between the A. gossypii genome and the $S$. cerevisiae genome have facilitated the assignment of potential functions to A. gossypii genes (Brachat et al., 2003; Dietrich et al., 2004). Moreover, other A. gossypii features have made the genetic manipulation of this fungus to be quite straightforward when comparing to other filamentous fungi, among which: highly efficient homologous recombination, which allows the use of PCR-based gene targeting tools (Steiner et al., 1995; Wendland et al., 2000); haploid nuclei and unicucleated spores (Steiner et al., 1995), which facilitate the isolation of stable homokaryotic strains; and ability to freely replicate plasmids containing $S$. cerevisiae autonomously replicating sequences (ARS) (Wright and Philippsen, 1991) and to regulate gene expression using S. cerevisiae promoters (Wendland et al., 2000; Kaufmann, 2009; Ribeiro et al., 2010; Magalhães et al., 2014), making possible the use of several tools already available to manipulate $S$. cerevisiae.

Although a wide range of molecular tools exist to genetically engineer $A$. gossypii, the number of selection markers in current use is limited. The absence of a known sexual cycle in $A$. gossypii has hindered the use of classical genetics approaches in this fungus (Wendland et al., 2011). Thus, the molecular characterization of $A$. gossypii genes has relied on reverse genetics approaches. However, only four marker genes are available for PCR-based gene targeting in A. gossypii, which has hampered the disruption and/or integration of multiple genes in the same strains. The existent markers are: (1) the S. cerevisiae LEU2 gene fused with its own promoter and terminator (Alberti-Segui et al., 2001), which can only be used as auxotrophic marker for gene manipulations in stable Agleu2 
strains; (2) the Escherichia coli $\mathrm{kan}^{r}$ gene fused to the $S$. cerevisiae TEF2 (ScTEF2) promoter and terminator (GEN3) (Wendland et al., 2000); (3) the Streptomyces noursei nat ${ }^{r}$ gene flanked by the S. cerevisiae PDC1 (SCPDC1) promoter and terminator (NATPS) (Hoepfner in Kaufmann, 2009); and (4) a $h_{y g}{ }^{r}$ gene fused to the promoter and terminator sequences of the A. gossypii LEU2 (AgLEU2) gene (Revuelta in Schlüpen, 2003; Jiménez et al., 2008). Because this last marker cassette contains sizeable homology regions to the AgLEU2 locus, when used for targeted engineering it can direct integration into the AgLEU2 locus rather than into the desirable target site.

The ability to rescue drug marker genes from the A. gossypii genome would therefore offer new perspectives in what concerns $A$. gossypii manipulation. Hence, the aim of this work was to develop a Cre-loxP-based system to remove and reuse selection markers in A. gossypii. To achieve this goal, several marker cassettes and Creexpressing plasmids were constructed and successfully used to excise the selection markers used to delete two model genes, the AgURA3 (AEL059W) gene and the predicted AgADE1 (AER221W) open reading frame (ORF). These genes were chosen because their deletion not only should provide easy phenotype identification as would result in new auxotrophic strains, further expanding the molecular toolbox available for A. gossypii.

\section{Materials and methods}

\subsection{Strains and media}

A. gossypii ATCC 10895 strain, kindly provided by Prof. P. Philippsen (University of Basel) was used as the background for all deletions. Strains generated in this study are listed in Table 1. A. gossypii strains were grown at $30^{\circ} \mathrm{C}$ on agar-solidified Ashbya full medium (AFM; $10 \mathrm{~g} / \mathrm{l}$ tryptone, $10 \mathrm{~g} / \mathrm{l}$ yeast extract, $20 \mathrm{~g} / \mathrm{l}$ glucose, $1 \mathrm{~g} / \mathrm{l}$ myo-inositol), containing $150 \mu \mathrm{g} / \mathrm{ml}$ geneticin (G418) (Sigma-Aldrich Química, Sintra, Portugal), $50 \mu \mathrm{g} / \mathrm{ml}$ nourseothricin (clonNAT) (WERNER BioAgents, Jena, Germany) or $5 \mu \mathrm{g} / \mathrm{ml}$ phleomycin (InvivoGen, Toulouse, France) for selection and maintenance of transformants. A. gossypii spores were prepared and stored as described by Ribeiro et al. (2010), with the exception that the mycelium was digested using $4 \mathrm{mg} / \mathrm{ml}$ Lysing Enzymes from
Trichoderma harzianum (Sigma-Aldrich Química, Sintra, Portugal). Agar-solidified Synthetic Complete (SC) medium (Sherman et al. 1986) buffered with $0.1 \mathrm{M}$ sodium-phosphate buffer ( $\mathrm{pH} 7.0$ ), lacking uracil and/or adenine, or supplemented with $5 \mathrm{mM}$ uridine, uracil and adenine (AppliChem GmbH, Darmstadt, Germany) was used for phenotype characterization. When indicated, 5-fluoroorotic acid (5-FOA) (Sigma-Aldrich Química, Sintra, Portugal) was added to agar-solidified AFM at a final concentration of $1 \mathrm{mg} / \mathrm{ml}$.

E. coli TOP10 (Invitrogen, Carlsbad, CA) served as plasmid host and was grown on agar-solidified LB medium (Sambrook and Russell, 2001) containing $100 \mu \mathrm{g} / \mathrm{ml}$ ampicillin (Sigma-Aldrich Química, Sintra, Portugal). Plasmids were maintained in a permanent culture at $-80^{\circ} \mathrm{C}$ in $2 \mathrm{ml}$ aliquots with $15 \%(\mathrm{w} / \mathrm{w})$ glycerol.

\subsection{Molecular methods}

DNA manipulations were carried out using standard procedures (Sambrook and Russell, 2001). Plasmid DNA was isolated from E. coli using the GenElute Plasmid Miniprep Kit (Sigma-Aldrich Química, Sintra, Portugal). PCR reactions were performed using NZYTaq DNA polymerase (NZYTech, Lisbon, Portugal) or Phusion High-Fidelity DNA polymerase (Finnzymes, Espoo, Finland). All primers used in this work are listed in Table 2 and were obtained from Frilabo (Maia, Portugal). PCR fragments were purified using the QIAquick PCR Purification Kit (QIAGEN, Hilden, Germany) and DNA fragments were recovered from agarose gels using the QIAquick Gel Extraction Kit (QIAGEN, Hilden, Germany). The restriction enzymes used in this study were purchased from New England BioLabs (Ipswich, USA) or NZYTech (Lisbon, Portugal). T4 DNA ligase was purchased from Promega (Madison, USA).

Transformation of $A$. gossypii was carried out as described by Wendland et al. (2000), using $30 \mu \mathrm{g}$ of DNA for integration of deletion cassettes, or $5 \mu \mathrm{g}$ of DNA for plasmid insertion. A. gossypii genomic DNA was extracted as follows. A piece of mycelium collected from the border of $A$. gossypii colonies was transferred to microcentrifuge tubes containing $200 \mu \mathrm{l}$ of autoclaved DNA extraction buffer ( $50 \mathrm{mM} \mathrm{NaCl}, 1 \mathrm{mM}$ EDTA pH 8.0, $10 \mathrm{mM}$ Tris- $\mathrm{HCl} \mathrm{pH}$ 8.0, and $0.5 \%(\mathrm{v} / \mathrm{v})$ Triton $\mathrm{X}-100)$ and quickly mixed by vortexing. $100 \mu \mathrm{l}$ of phenol:chloroform:isoamyl alcohol (25:24:1) were sub-

Table 1

Strains and plasmids used in this study.

\begin{tabular}{|c|c|c|}
\hline $\begin{array}{l}\text { Strain or } \\
\text { plasmid }\end{array}$ & Relevant feature(s) & Reference \\
\hline \multicolumn{3}{|l|}{ A. gossypii strains } \\
\hline ATCC 10895 & Parent strain & Prof. P. Philippsen \\
\hline Agura3GEN3 & Derived from ATCC 10895; Agura3 $\Delta:$ :loxP-GEN3-loxP & This work \\
\hline Agade1NATPS & Derived from ATCC 10895; Agade1A::loxP-NATPS-loxP & This work \\
\hline Agura3 & Derived from Agura3GEN3; Agura3 $\Delta:: l o x P$ & This work \\
\hline Agade1 & Derived from Agade1NATPS; Agade1 $\Delta:: l o x P$ & This work \\
\hline Agura3ade1GEN3 & Derived from Agura3; Agura3 $\Delta::$ loxP Agade1 1 ::loxP-GEN3-loxP & This work \\
\hline Agura3ade1 & 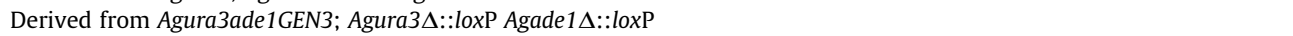 & This work \\
\hline \multicolumn{3}{|l|}{ Plasmids } \\
\hline pGEN3 & $\begin{array}{l}\text { GEN3 cassette conferring resistance to G418: comprises the E. coli } \text { kan }^{r} \text { gene from transposon Tn903 under the control of } \\
\text { the S. cerevisiae TEF2 promoter and terminator; ampicilin resistance }\end{array}$ & Wendland et al. (2000) \\
\hline pUG66 & $\begin{array}{l}\text { bleMX cassette conferring resistance to phleomycin: comprises the bacterial ble }{ }^{r} \text { gene from transposon } \mathrm{Tn} 5 \text { under the } \\
\text { control of the A. gossypii TEF promoter and terminator flanked by two loxP sequences; ampicillin resistance }\end{array}$ & Gueldener et al. (2002) \\
\hline pUC19NATPS & $\begin{array}{l}\text { NATPS cassette conferring resistance to clonNAT: comprises the Streptomyces noursei nat1 gene flanked by the S. cerevisiae } \\
\text { PDC1 promoter and terminator; ampicillin resistance }\end{array}$ & $\begin{array}{l}\text { D. Hoepfner in } \\
\text { Kaufmann (2009) }\end{array}$ \\
\hline pUGGEN3 & Derivative of pUG66 with the GEN3 cassette from pGEN3 instead of the bleMX cassette & This work \\
\hline pUGNATPS & Derivative of pUG66 with the NATPS cassette from pUC19NATPS instead of the bleMX cassette & This work \\
\hline pUGBLE3 & Derivative of pUGGEN3 with the ble ${ }^{r}$ gene from pUG66 under the control of the S. cerevisiae TEF2 promoter and terminator & Ribeiro et al. (2013) \\
\hline pNatCre & $\begin{array}{l}\text { natMX marker conferring resistance to clonNAT; comprises the nat } 1 \text { gene under the control of the A. gossypii TEF promoter } \\
\text { and terminator; bacteriophage P1 cre gene under the control of the S. cerevisiae GAL1 promoter and CYC1 terminator; } \\
\text { ARSH-CEN6 replication sequences from S. cerevisiae; ampicillin resistance }\end{array}$ & $\begin{array}{l}\text { Steensma and Ter } \\
\text { Linde (2001) }\end{array}$ \\
\hline pAgNatCre & Derivative of pNatCre with the cre gene under the control of the $S$. cerevisiae ADH2 promoter & This work \\
\hline pAgBleCre & Derivative of pAgNatCre with the bleMX marker from pUG66 in place of the natMX cassette & This work \\
\hline
\end{tabular}


Table 2

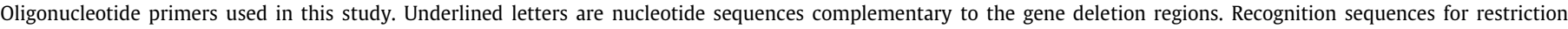
enzymes are in bold.

\begin{tabular}{|c|c|}
\hline Name & Sequence $\left(5^{\prime}-3^{\prime}\right)$ \\
\hline G1 & GCTAGGGATAACAGGGTAAT \\
\hline G2 & CCGCTCGAGGATCTGATGAGGCCGTCT \\
\hline G3 & GATGGTGTAGGCTCCACATCACAGTAAGCATTTGTATAAGGCTGATCACATAGGGTGCTACCGACCCTAGCCATTGCCACGCAGGTCGACAACCCTTAAT \\
\hline G4 & GGGCATATAATTACAAAAAGGTTGCTGAGATGGTAATCGGCGGTGGAATCCGCCTATATACTGTGGGCGCCAGCGATAGAGCATAGGCCACTAGTGGATC \\
\hline U1 & CCATTGCCACATGTCAACGAAATC \\
\hline U2 & GTGTTGGATGCATGCTACTGAAC \\
\hline G5 & TCGCAGACCGATACCAGGATC \\
\hline U3 & AATCGCTCGAGCAACTCATTGG \\
\hline N1 & GAAGATCTTCCCTGCAGAACCGTTACGGTA \\
\hline $\mathrm{N} 2$ & CCGCTCGAGCCTGCAGCCAAACAGTGTT \\
\hline N3 & CCAGGCAGCGCGAGCTTGACTCTGGTCTATGGCCAATTTAACAGCCCCATATAGACAAGATATATATAGAGAGATAGCAGGCAGGTCGACAACCCTTAAT \\
\hline N4 & САCATCTCCATTTATCACTTAAAATTATTTAACATTTTAAAAGTACATCATTTTACCATTTCGCGCCTGTAAGTGCTTCGGCATAGGCCACTAGTGGATC \\
\hline A1 & CAATGCGTCTCGAGGAATTTGCTCG \\
\hline A2 & TCGGGTGACGTTAGCGCATCA \\
\hline A3 & AGAAGCAAGCGAGCGACTACG \\
\hline N5 & GATTCGTCGTCCGATTCGTC \\
\hline AH1 & CGAGCTCGCAAAGGGGCAAAACGTAG \\
\hline $\mathrm{AH} 2$ & CGGAATTCCGTGAGTTTCTGGAATAGACATTG \\
\hline ScTEFt_EcoRI & CGGAATTCTCGATGAGTTTTTCTAAGAG \\
\hline ScTEFp_NcoI & TCTATTCCATGGTAATTATAGTTCGTTGACCG \\
\hline Ble & GCAGGTCGACAACCCTTAAT \\
\hline Ble_EcoRI & CGGAATTCTCATGAGATGCCTGCAAG \\
\hline qPCRAgACT1_F & TCGTTGCCACACGCCATTT \\
\hline qPCRAgACT1_R & AAAGGAGTAGCCACGTTCCGATAG \\
\hline qPCRCRE_F & CGTACTGACGGTGGGAGAATGTTA \\
\hline qPCRCRE_R & TCCATCGCTCGACCAGTTTAGT \\
\hline
\end{tabular}

sequently added, followed by four vortexing cycles of $10 \mathrm{~s}$ each, with intercalary cooling on ice. After centrifugation for 15 min at maximum speed, $20 \mu \mathrm{l}$ of the upper aqueous phase were diluted with $80 \mu \mathrm{l}$ of water. 1-2 $\mu \mathrm{l}$ of these DNA samples were used as template for PCR amplification.

\subsection{DNA constructs}

The deletion cassettes loxP-GEN3-loxP and loxP-NATPS-loxP were constructed as follows. The GEN3 cassette was amplified from pGEN3 (Wendland et al., 2000) using primers G1 and G2 (Table 2) and the NATPS cassette amplified from pUC19NATPS (Hoepfner in Kaufmann, 2009) with primers N1 and N2 (Table 2). Both fragments were digested with BglII and XhoI, and cloned between the loxP sequences of BglII/Xhol digested pUG66 (Gueldener et al., 2002) to create pUGGEN3 and pUGNATPS, respectively. A loxPBLE3-loxP cassette was also generated as described in Ribeiro et al. (2013). Briefly, the entire pUGGEN3 sequence, with the exception of the $\mathrm{kan}^{r}$ gene, was amplified with the primers ScTEFt_EcoRI and ScTEFp_NcoI (Table 2) and the $b l e^{r}$ gene obtained by PCR from plasmid pUG66 with the primers Ble and Ble_EcoRI (Table 2). Both PCR fragments were digested with EcoRI and NcoI and ligated to create pUGBLE3.

For deletion of the AgURA3 (AELO59W) gene, the loxP-GEN3-loxP cassette was obtained by PCR from pUGGEN3 with primers G3 and G4 (Table 2). For deletion of the AgADE1 (AER221W) ORF, either loxP-GEN3-loxP or loxP-NATPS-loxP cassettes were obtained by PCR from pUGGEN3 or pUGNATPS, respectively, with primers N3 and N4 (Table 2). The amplified deletion modules comprised the resistance cassettes flanked by loxp sequences and 80 bp guide sequences with homology to the $5^{\prime}$ and $3^{\prime}$ untranslated regions (UTRs) of the target loci, to ensure recombination in the correct location and direction in the genome.

Plasmids pAgNatCre and pAgBleCre were constructed from pNatCre (Steensma and Ter Linde, 2001), a shuttle vector that harbors ARSH-CEN6 elements from S. cerevisiae, the natMX selective marker and the bacteriophage P1 cre gene flanked by the
S. cerevisiae GAL1 (ScGAL1) promoter and CYC1 (ScCYC1) terminator. For that, a $599 \mathrm{bp}$ fragment containing the $S$. cerevisiae $A D H 2$ (ScADH2) promoter, from position $872,712-873,310$ on S. cerevisiae chromosome XIII, was amplified from the strain CEN.PK 113-7D genomic DNA with primers AH1 and AH2 (Table 2). The amplified fragment was digested with the restriction enzymes SacI and EcoRI and cloned between the SacI/EcoRI sites of pNatCre in place of the ScGAL1 promoter. This new plasmid was named pAgNatCre. The pAgBleCre plasmid was obtained by the substitution of the natMX cassette from pAgNAtCre with the bleMX cassette from pUG66 with the restriction enzymes BglII and SacI. All plasmids used and generated in this study are listed in Table 1.

\subsection{Isolation of homokaryotic mutants and diagnostic PCR}

Clonal selection of $A$. gossypii homokaryotic mutants was performed through the isolation of single uninucleated haploid spores from primary heterokaryotic transformants. For that, different dilutions of spores harvested from primary transformants were spread onto selective AFM plates with a guide line drawn in the bottom. After germination for approximately $24 \mathrm{~h}$, plates were visually analyzed with a Nikon Diaphot 300 inverted microscope (Sony, Portugal) and single germlings were marked, isolated with a sterile scalpel and transferred to new selective AFM plates. The absence of the gene of interest was tested via diagnostic PCR.

PCR reactions were carried out in $25 \mu \mathrm{l}$ reaction mixtures with 1-2 $\mu$ l DNA template, $0.35 \mu \mathrm{M}$ of each primer, $200 \mu \mathrm{M}$ dNTP mix, $0.05 \mathrm{U} / \mu \mathrm{l}$ NZYTaq and NZYTaq buffer with $1.5 \mathrm{mM} \mathrm{MgCl}_{2}$ provided by the manufacturer (NZYTech, Lisbon, Portugal). PCR conditions were: $5 \mathrm{~min}$ at $95^{\circ} \mathrm{C}, 35$ cycles of $95^{\circ} \mathrm{C}$ for $45 \mathrm{~s}, 50{ }^{\circ} \mathrm{C}$ for $2 \mathrm{~min}$ (with increments of $0.3^{\circ} \mathrm{C}$ per cycle), $72{ }^{\circ} \mathrm{C}$ for $1 \mathrm{~min} 30 \mathrm{~s}$, and a final extension at $72{ }^{\circ} \mathrm{C}$ for $5 \mathrm{~min}$. Correct integration of the loxPGEN3-loxP cassette in the AgURA3 and AgADE1 loci was verified using the primer pairs $\mathrm{U} 1 / \mathrm{U} 2, \mathrm{G} 5 / \mathrm{U} 2$ and $\mathrm{U} 3 / \mathrm{U} 2$ (Table 2), and A1/A2, G5/A3 and A1/A3 (Table 2), respectively. Integration of the loxP-NATPS-loxP cassette in the AgADE1 locus was confirmed using the primer pairs A1/A2, A1/N5 and A1/A3 (Table 2). 
A
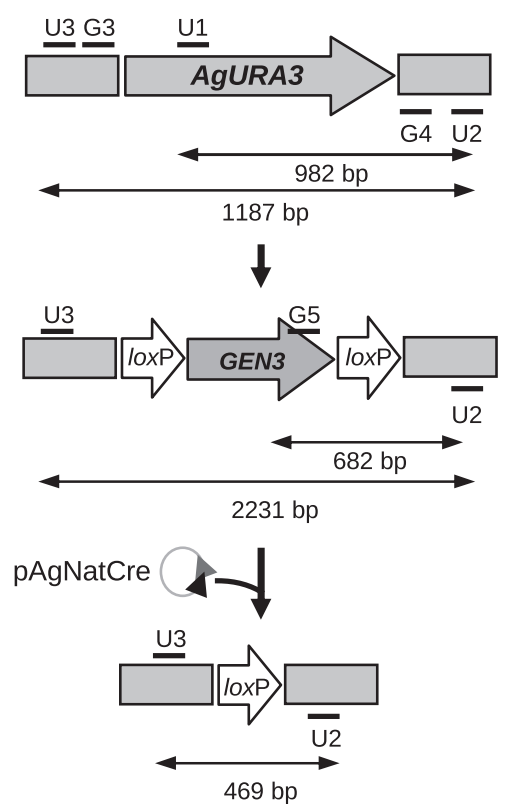

B

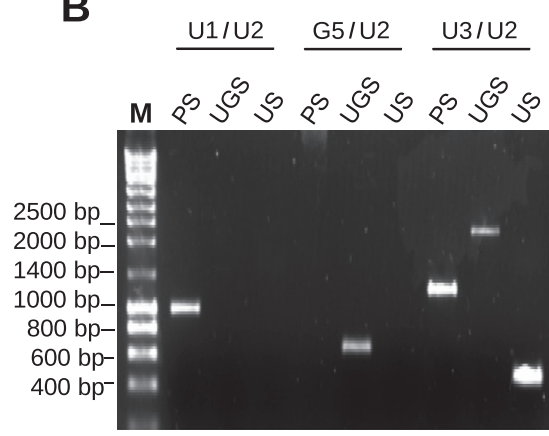

C
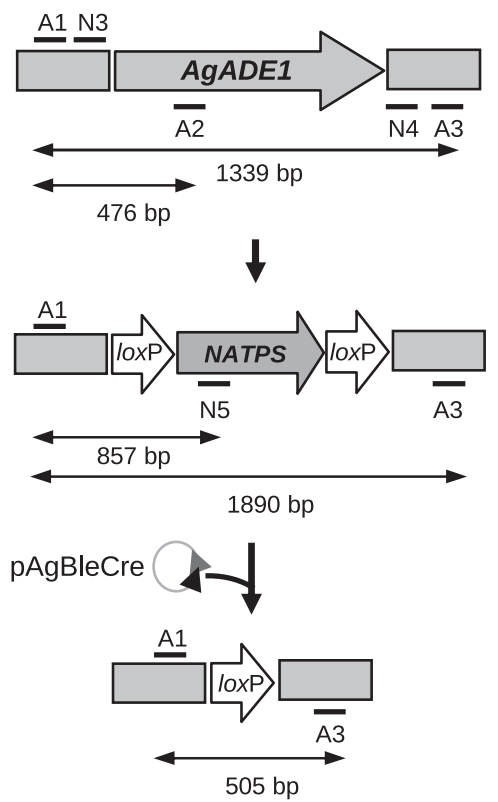

D

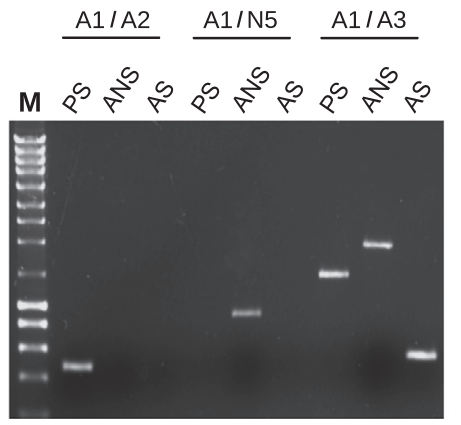

E
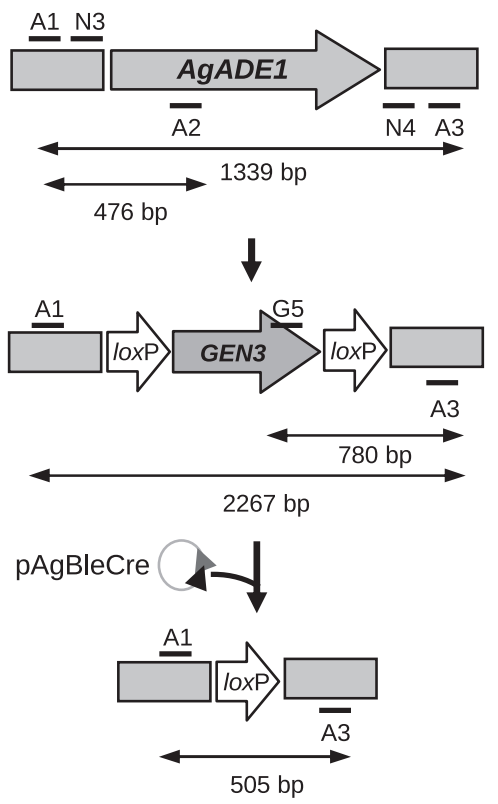

$\mathbf{F}$

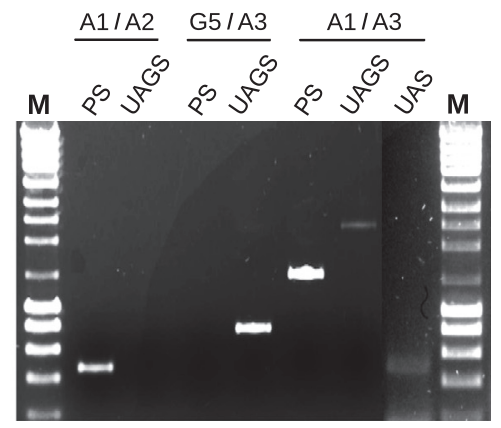

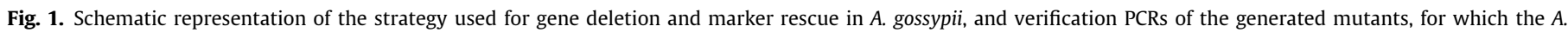

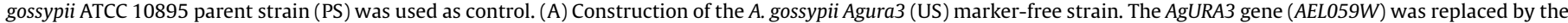

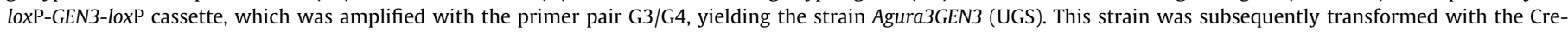

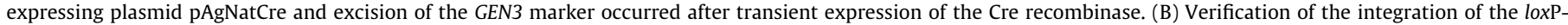

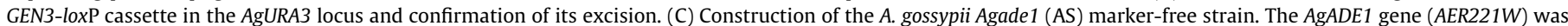

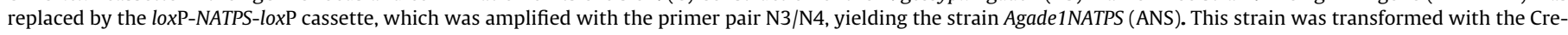

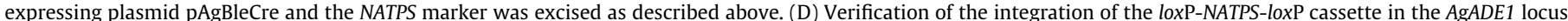

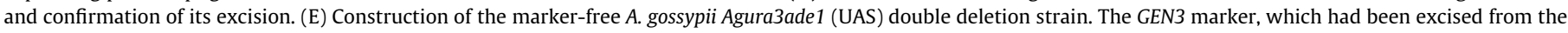

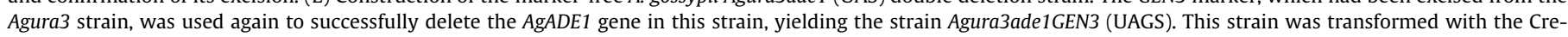

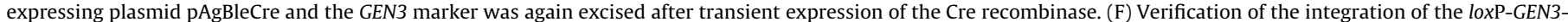
loxP cassette in the AgADE1 locus of the Agura3 strain and confirmation of its excision.

The positions of the primers and expected PCR amplicon sizes are given in Fig. 1.

\subsection{Cre recombinase expression and marker cassette excision}

For excision of the loxP-flanked marker cassettes, the mutant Agura3GEN3 was transformed with the Cre-expressing plasmid pAgNatCre and the Agade1NATPS and Agura3ade1GEN3 mutants with the plasmid pAgBleCre. The transformants were selected on agar-solidified AFM containing $50 \mathrm{~g} / \mathrm{l}$ glucose and $50 \mu \mathrm{g} / \mathrm{ml}$ of clonNAT or $5 \mu \mathrm{g} / \mathrm{ml}$ of phleomycin, depending on the resistance conferred by the plasmid. To express the Cre recombinase, positive transformant colonies were grown for 1 week on agar-solidified AFM containing $10 \mathrm{~g} / \mathrm{l}$ ethanol as sole carbon source plus the corresponding antibiotic selection. Simultaneously, the same transformants were also grown on selective AFM containing $50 \mathrm{~g} / \mathrm{l}$ glucose, to test whether the expression of the Cre recombinase would be different in these conditions. Subsequently, spores harvested from these plates were spread onto agar-solidified AFM without selection, for plasmid loss. Small patches from the edge of each new mycelial colony were transferred to agar-solidified AFM containing G418 and/or clonNAT (depending on the resistance conferred by the deletion cassette used) and agar-solidified AFM without antibiotic. No growth in plates containing antibiotic indicated successful excision of the marker cassettes through recombination of the loxP sequences. The removal of the loxP-flanked marker cassettes was further confirmed by PCR and stable homokaryotic mutants free of exogenous markers were isolated as described above.

\subsubsection{Quantitative PCR}

For the expression analysis of the cre recombinase, mycelia from a colony of Agura3GEN3 transformed with the Cre-expressing plasmid pAgNatCre were harvested (in triplicate) after 4 days of 
growth on agar-solidified AFM containing $50 \mu \mathrm{g} / \mathrm{ml}$ clonNAT and either $10 \mathrm{~g} / \mathrm{l}$ ethanol or $50 \mathrm{~g} / \mathrm{l}$ glucose. Mycelia from untransformed Agura3GEN3 grown for 4 days on agar-solidified AFM containing $10 \mathrm{~g} / \mathrm{l}$ ethanol or $50 \mathrm{~g} / \mathrm{l}$ glucose were also harvested in triplicate. Total RNA was extracted with the RNeasy Plant Mini kit (QIAGEN, Hilden, Germany) using the manufacturer's instructions for filamentous fungi and its concentration and purity was determined as previously described (Aguiar et al., 2014). After treatment with DNase I (Thermo Fisher Scientific, Loures, Portugal), $1 \mu \mathrm{g}$ of total RNA was reverse transcribed using the NZY First-Strand cDNA Synthesis Kit (NZYTech, Lisbon, Portugal). Quantitative PCR (qPCR) analyses were performed on a Bio-Rad (Hercules, CA) CFX 96 real-time PCR system as previously described (Aguiar et al., 2014), with the primer pairs shown in Table 2. Each cDNA (1:10) sample was analyzed in duplicate and the average cycle threshold was calculated. Relative expression levels were determined with the $2^{-\Delta \Delta C T}$ (Livak) method, using the experimentally determined amplification efficiency of the primers. The cre expression levels were normalized to the expression level of actin $(A g A C T 1)$ for each strain in each condition.

\section{Results}

\subsection{Constructs generated for marker recycling in A. gossypii}

New disruption cassettes for use in A. gossypii, which could be excised after being used to screen for a genetic modification and then reused to produce multiple gene deletions in the same strain, were constructed using as backbone the plasmid pUG66 (Gueldener et al., 2002). The loxP-GEN3-loxP and loxP-NAPS-loxP cassettes were obtained by replacing the bleMX unit located between the two loxP sites in plasmid pUG66 with the GEN3 and NATPS units from plasmids pGEN3 (Wendland et al., 2000) and pUC19NATPS (Hoepfner in Kaufmann, 2009), respectively, yielding plasmids pUGGEN3 and pUGNATPS (Fig. 2). The $b l e^{r}$ gene from transposon Tn5, which confers resistance to phlemoycin (Gatignol et al., 1987), was used to create the new heterologous dominant drug resistance marker cassette loxP-BLE3-loxP, as described in Ribeiro et al. (2013). The $b l e^{r}$ gene was isolated by
PCR from plasmid pUG66 and placed between the SCTEF2 promoter and terminator in plasmid pUGGEN3, yielding plasmid pUGBLE3 (Fig. 2).

New plasmids for transient expression of the Cre recombinase in A. gossypii were constructed using as backbone the replicative ARSH-CEN6 plasmid pNatCre (Steensma and Ter Linde, 2001). This plasmid harbors a Cre expression cassette where the cre gene is flanked by the ScGAL1 promoter and ScCYC1 terminator sequences. Several GAL pathway genes are absent in the A. gossypii genome (Hittinger et al., 2004), including the ScGAL1 homolog, hampering its growth on galactose. Hence, the $S C A D H 2$ promoter was used instead to mediate the Cre recombinase expression in A. gossypii. In the plasmid pAgNatCre, the $467 \mathrm{bp} \mathrm{ScGAL1}$ promoter was therefore substituted with the $599 \mathrm{bp} S \mathrm{~S} A D H 2$ promoter. Plasmid pAgBleCre was obtained by substituting the natMX drug marker in pAgNatCre with the bleMX expression cassette from plasmid pUG66. The usable range of phleomycin concentrations for selection of A. gossypii phleomycin-resistant colonies was determined and the optimal concentration for selection was found to be $5 \mu \mathrm{g} / \mathrm{ml}$ phleomycin (in agar-solidified AFM).

\subsection{Deletion of the AgURA3 gene and generation of an uracil auxotrophic strain}

As a model to test the functionality of the Cre-loxP system in $A$. gossypii, the entire coding region of the AgURA3 (AELO59W) gene was deleted from the A. gossypii genome using the loxP-GEN3-loxP cassette flanked by 80 bp guide sequences with homology to the AgURA3 $5^{\prime}$ and $3^{\prime}$ UTRs. Homologous integration of this deletion module within the AgURA3 locus resulted in the replacement of AgURA3 by the loxP-GEN3-loxP cassette with its transcriptional orientation corresponding to that of the deleted gene (Fig. 1A). Of the primary transformants randomly screened 53\% were heterokaryotic, containing nuclei with both wild type and null alleles of AgURA3 (Fig. 2). After a single spore purification step, diagnostic PCR indicated the sole presence of the mutant allele in the Agura3GEN3 strain (Fig. 1B).

Deletion of the AgURA3 gene caused uridine/uracil auxotrophy, being the homokaryotic Agura3GEN3 strain unable to grow on

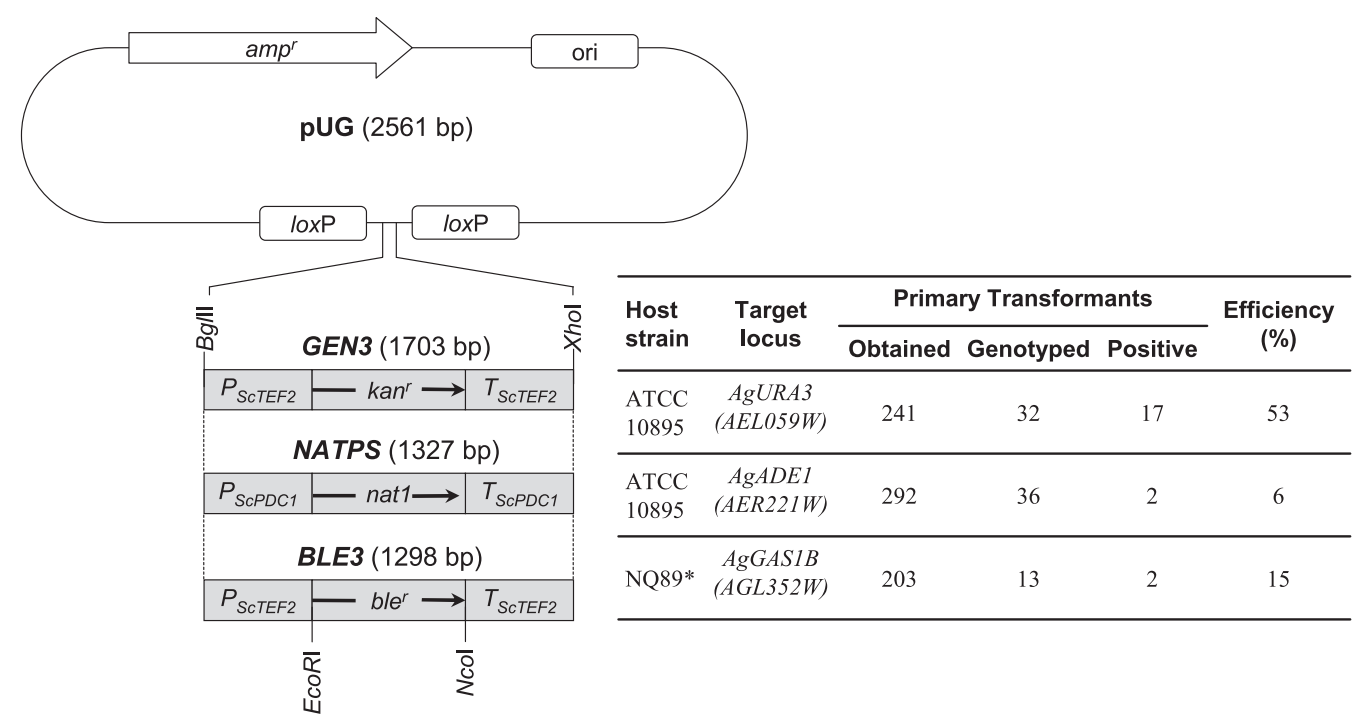

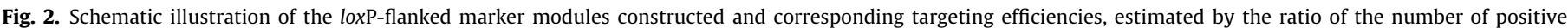

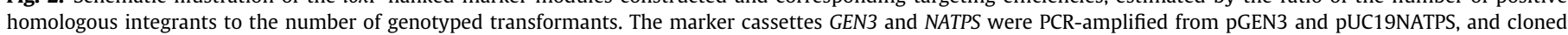

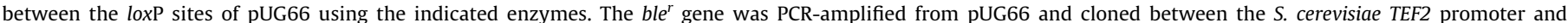

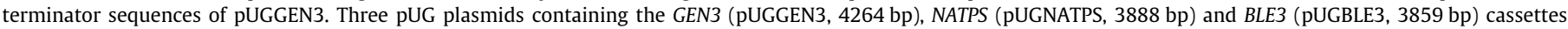
flanked by loxP-sequences were obtained. "Ribeiro et al. (2013). 


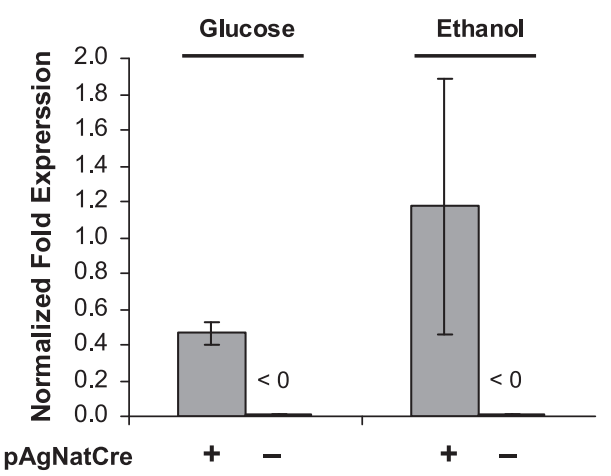

Fig. 3. $q P C R$ analysis of the cre expression in Agura3GEN3 transformed (+) or not (-) with the Cre-expressing plasmid pAgNatCre. The strains were grown for 4 days on agar-solidified AFM containing $50 \mathrm{~g} / \mathrm{l}$ glucose or $10 \mathrm{~g} / \mathrm{l}$ ethanol. The cre expression levels were normalized to the expression level of actin (AgACT1). Data represents the mean \pm SD of three biological replicates.

defined medium lacking uridine and uracil (Fig. 4). When the medium was supplemented with uridine and uracil the Agura3GEN3 strain grew similarly to the parent strain, which did not happen when the medium was supplemented with uracil alone (Fig. 4). Moreover, contrary to the parent strain, the Agura3GEN3 strain was able to grow on agar-solidified AFM containing $1 \mathrm{mg} / \mathrm{ml}$ 5-FOA (Fig. 4).

\subsection{Transient expression of Cre recombinase and marker rescue}

The Agura3GEN3 strain was transformed with the plasmid pAgNatCre to allow the transient expression of Cre recombinase. Transformants were selected on rich AFM containing clonNAT and $50 \mathrm{~g} / \mathrm{l}$ glucose. Spores harvested from a randomly selected transformant colony were grown for 1 week on agar-solidified AFM containing clonNAT and $10 \mathrm{~g} / \mathrm{l}$ ethanol as sole carbon source. The spores collected after this period were spread onto non-selective AFM, to promote plasmid loss, and the sensitivity of the resulting colonies to clonNAT and G418 was tested. Of the 39 mycelial colonies screened none was able to grow on AFM containing clonNAT, indicating that all had lost the plasmid that contained the nat$M X$ resistance marker after only one passage in non-selective conditions. Thirty-six colonies were also unable to grow on AFM containing G418, from which the GEN3 marker had been looped out and lost. After a single spore purification step, the excision of the GEN3 cassette from the Agura3 locus was confirmed by PCR (Fig. 1B). This homokaryotic strain free of exogenous marker genes was named Agura3.

To test whether the excision of the GEN3 cassette also occurred in glucose-containing medium, spores from the same randomly selected transformant colony were simultaneously grown on agar-solidified AFM containing clonNAT and $50 \mathrm{~g} / \mathrm{l}$ glucose. In this case, 42 of the 49 mycelial colonies screened also lost their resistance to G418. Moreover, no statistically significant differences were found in the cre transcript levels between these conditions $(p=0.159)$, although a tendency was observed for lower expression of the Cre recombinase in glucose-containing medium (Fig. 3).

To assess the occurrence of background excision (i.e. Cre recombinase-independent excision), the untransformed Agura3GEN3 strain was also grown for 1 week on agar-solidified AFM containing either $10 \mathrm{~g} / \mathrm{l}$ ethanol or $50 \mathrm{~g} / \mathrm{l}$ glucose. Of the 63 mycelial colonies screened for each condition none was sensitive to G418. Moreover, the transcript levels of the cre gene in this strain were undetectable (Fig. 3). Altogether, these data indicate that the excision of the GEN3 cassette was dependent on the expression of the Cre recombinase.

\subsection{Deletion of the AgADE1 gene and generation of a stable adenine} auxotrophic strain

To demonstrate the versatility of the developed Cre-loxP system, the predicted $A g A D E 1$ ( $A E R 221 W$ ) ORF was deleted using the loxP-NATPS-loxP cassette, with a targeting efficiency of $6 \%$ (Fig. 2). The correct integration of this deletion cassette was verified by PCR, as shown in Fig. 1D, and the resulting homokaryotic strain was named Agade1NATPS.

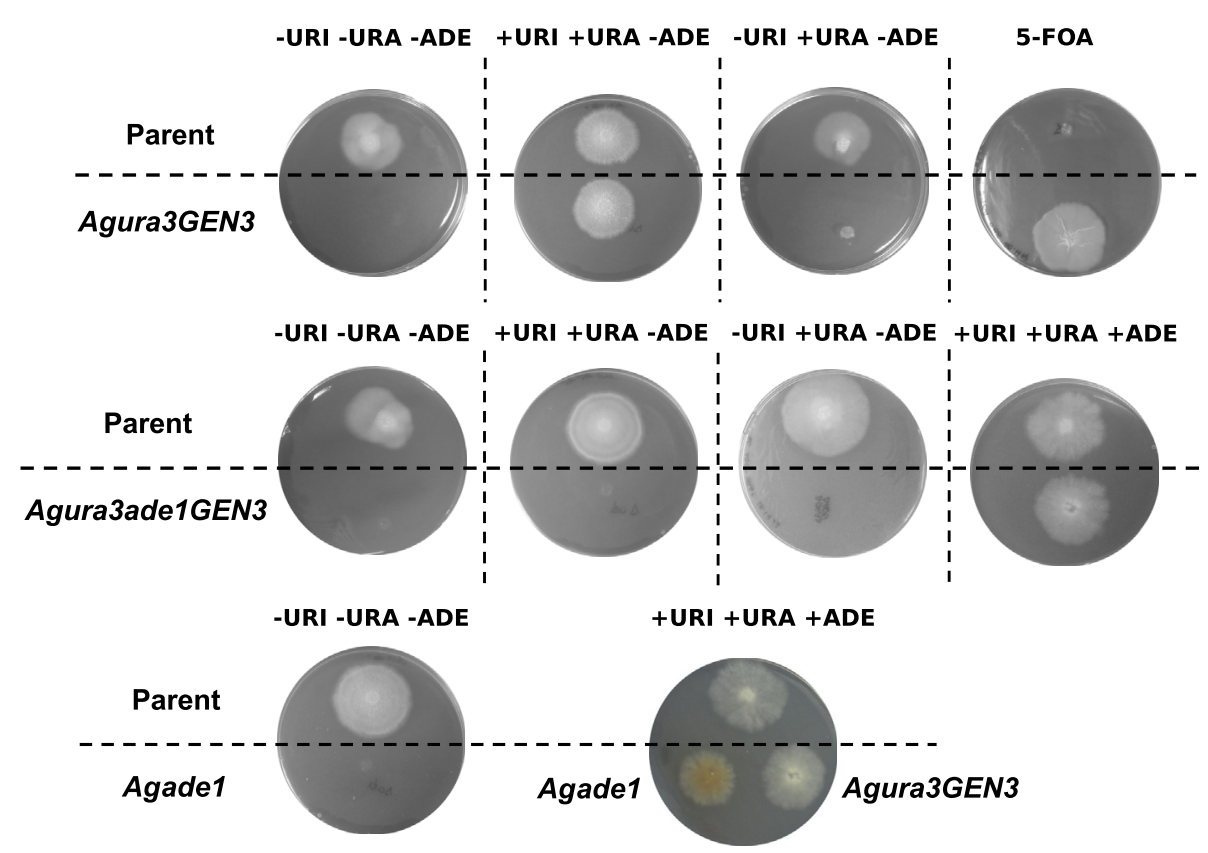

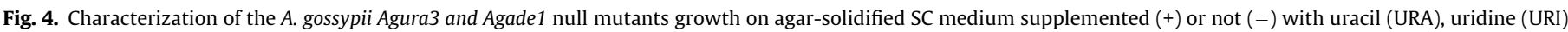

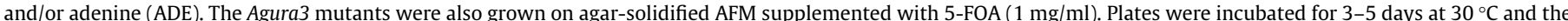

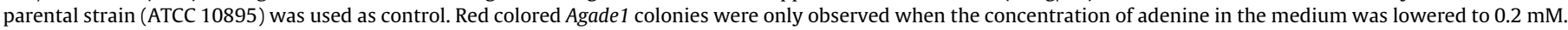
(For interpretation of the references to color in this figure legend, the reader is referred to the web version of this article.) 
This strain was then transformed with the Cre-expressing plasmid pAgBleCre, transformants were selected on AFM containing phleomycin and $50 \mathrm{~g} / \mathrm{l}$ glucose, and Cre recombinase was transiently expressed as described above. Following this strategy, the loxP-flanked NATPS cassette could be looped out of the chromosomal DNA of Agade1NATPS transformant colonies. After a single spore purification step, a homokaryotic Agade1 strain free of marker genes (Fig. 1D) and auxotrophic for adenine (Fig. 4) was obtained.

\subsection{Demonstration of marker recycling by deletion of the AgADE1 gene from the genome of the Agura3 strain}

To demonstrate the successful recycling of the GEN3 marker, the $A g A D E 1$ ORF was also deleted from the genome of the Agura3 strain using the loxP-GEN3-loxP cassette previously used to delete the AgURA3 ORF, which was flanked by 80 bp guide sequences with homology to the AgADE1 5' and 3' UTRs. The correct integration of the deletion cassette in the AgADE1 locus was verified by PCR as shown in Fig. 1F. Homokaryotic Agura3ade1GEN3 mutants were obtained after a single spore purification step (Fig. 1F).

Physiological characterization of this mutant revealed that it was auxotrophic for both uridine/uracil and adenine (Fig. 4). This strain also continued to be resistant to 5 -FOA $(1 \mathrm{mg} / \mathrm{ml})$.

To excise again the GEN3 cassette from this strain, it was transformed with the Cre-expressing plasmid pAgBleCre for transient expression of the Cre recombinase, as described in Section 2. Of the 40 mycelial colonies screened after the transient expression protocol, 36 were unable to grow on AFM containing G418, suggesting that the GEN3 marker had been lost again. After a single spore purification step, the excision of the GEN3 cassette from the Agade1 locus was confirmed by PCR (Fig. 1F). This homokaryotic strain free of exogenous marker genes was named Agura3ade1.

\section{Discussion}

Here, the Cre-loxP recombination system of the bacteriophage P1 was adapted to genetically engineer $A$. gossypii in a targeted way and allow the removal and reuse of the selection markers used during the process. Three heterologous recyclable drug marker cassettes and two Cre recombinase expression vectors for use in A. gossypii were created and successfully used to generate stable Agura3 and Agade1 auxotrophic strains free of exogenous selectable markers.

The AgURA3 gene encodes the orotidine-5'-phosphate decarboxylase involved in the biosynthesis of pyrimidines (Pompejus et al., 1999) and its deletion caused uridine/uracil auxotrophy, and conferred resistance to 5-FOA. In the parent strain, growth was hampered by the presence of 5-FOA in the medium, which indicates that the AgUra3p converts 5-FOA into 5-fluorouracil, a toxic compound that causes cell death (Boeke et al., 1984). Therefore, the use of the AgURA3 gene as a bidirectionally selectable marker (conferring uridine/uracil prototrophy and 5-FOA sensitivity) may be considered in future A. gossypii engineering projects. However, it is worth noting that in previous reports neither the $S$. cerevisiae URA3 nor the E. coli pyrF genes were able to complement uracil auxotrophy in A. gossypii Agura3 mutants (Pompejus et al., 1999). Therefore, efforts are now being made to develop an expression cassette that can complement the AgURA3 auxotrophy.

Based on the analysis of the annotated genome sequence of $A$. gossypii (http://agd.vital-it.ch; Gattiker et al., 2007), the AER221W ORF (AgADE1) was found to be homologous to the $S$. cerevisiae $A D E 1$ gene. In $S$. cerevisiae, this gene encodes the $N$-succinyl-5aminoimidazole-4-carboxamide ribotide (SAICAR) synthetase involved in the purine biosynthetic pathway and its deletion causes adenine auxotrophy, and vacuolar accumulation of red-colored purine precursors when the exogenous adenine supplied is limited (Fischer, 1969). Both these phenotypes were also observed in the $A$. gossypii Agade1 null mutants (Fig. 4). Previously, the deletion of the $A g A D E 4$ gene had also been shown to cause adenine auxotrophy in A. gossypii (Jiménez et al., 2005), but not the red pigmentation phenotype characteristic of ade1 and ade2 mutants (Fischer, 1969).

For $S$. cerevisiae, several recyclable marker cassettes and Cre-expressing plasmids are available (Güldener et al., 1996; Steensma and Ter Linde, 2001; Gueldener et al., 2002). Unfortunately, these cannot be used in A. gossypii for several reasons. First, most of the available cassettes are not heterologous to the A. gossypii genome, as they contain the A. gossypii TEF promoter and terminator sequences. Second, the heterologous cassettes available are auxotrophic markers, therefore requiring the existence of auxotrophic strains. Third, the cre gene present in the available plasmids is under the regulation of the ScGAL1 promoter (Steensma and Ter Linde, 2001; Gueldener et al., 2002), which cannot be used in A. gossypii because this fungus lacks pathways for the utilization of galactose in its genome (Dünkler and Wendland, 2007).

Therefore, the heterologous GEN3 and NATPS markers widely used for PCR-based gene targeting in A. gossypii were adapted to contain loxP flanking sequences and successfully used to select Agura3 and Agade1 mutants. A new loxP-flanked heterologous drug resistance cassette was also constructed, which comprises the ble ${ }^{r}$ gene from transposon Tn5 fused to the ScTEF2 promoter and terminator (BLE3). This marker cassette renders resistance to phleomycin, a copper-containing antibiotic that acts by interfering with DNA synthesis (Gatignol et al., 1987). Although the BLE3 marker was not used to delete any of the genes presented here, it was successfully used to generate and select A. gossypii Aggas1agas $1 b$ null mutants (Ribeiro et al., 2013), with a targeting efficiency of approximately $15 \%$ (Fig. 3).

In the generated Cre-expressing plasmids, the ScGAL1 promoter was substituted by the $S C A D H 2$ promoter to allow transient expression of the Cre recombinase in A. gossypii. In S. cerevisiae, the regulation of the $S C A D H 2$ promoter is well studied, being its expression repressed several hundred-fold in the presence of glucose and activated when the glucose is depleted from the medium (Lee and DaSilva, 2005). In A. gossypii, as this promoter had never been used before little was known about its functionality, although previous observations had shown that the transcription of the A. gossypii SCADH2 homolog (AAR084W) was also repressed by the presence of glucose in the medium (our microarray data). In this study, the expression of the Cre recombinase on agar-solidified AFM containing glucose was slightly lower than when ethanol was used instead as carbon source, but this difference was not statistically significant (Fig. 3). On the other hand, no cre transcripts were detected in strains lacking Cre-expressing plasmids, neither could be detected Cre recombinase-independent excisions of the marker cassettes. The constructed plasmids thus allowed the transient expression of the Cre recombinase, necessary to generate the mutant strains free of exogenous markers, which could be stopped after one subculturing step in unselective medium for plasmid loss.

In summary, the method here presented for multiple gene disruption and/or integration in $A$. gossypii offers advantages over the existing methods by enabling the creation of mutants entirely free of foreign genes and allowing the reuse of selection markers. The set of disruption cassettes and plasmids constructed greatly expand the possibilities for genetically engineer $A$. gossypii, being these suitable for use in both laboratorial and industrial strains, as they do not require any predetermined genetic background. Furthermore, since the number of stable A. gossypii auxotrophic strains currently available is limited, the strains generated here are envisaged to be useful for future genetic engineering projects. Efforts are now being made to develop a selection system based on the complementation of these auxotrophic mutants. 


\section{Acknowledgments}

This work was supported by Fundação para a Ciência e a Tecnologia, Portugal, through Project AshByofactory (PTDC/ EBB-EBI/101985/2008 - FCOMP-01-0124-FEDER-009701), Project RECI/BBB-EBI/0179/2012 - FCOMP-01-0124-FEDER-027462, Strategic Project PEst-OE/EQB/LA0023/2013, Project BioInd (NORTE-07-0124-FEDER-000028) co-funded by the Programa Operacional Regional do Norte (ON.2 - O Novo Norte), QREN, FEDER, and MIT-Portugal Program PhD Grant SFRH/BD/39112/2007 to Tatiana Q. Aguiar.

\section{References}

Aguiar, T.Q., Dinis, C., Magalhães, F., Oliveira, C., Wiebe, M.G., Penttilä, M., Domingues, L., 2014. Molecular and functional characterization of an invertase secreted by Ashbya gossypii. Mol. Biotechnol. 56, 524-534.

Alberti-Segui, C., Dietrich, F., Altmann-Jöhl, R., Hoepfner, D., Phillipsen, P., 2001. Cytoplasmic dynein is required to oppose the force that moves nuclei towards the hyphal tip in the filamentous ascomycete Ashbya gossypii. J. Cell Sci. 114, 975-986.

Ashby, S.F., Nowell, W., 1926. The fungi of stigmatomycosis. Ann. Bot. 40, 69-84.

Boeke, J.D., LaCroute, F., Fink, G.R., 1984. A positive selection for mutants lacking orotidine-5'-phosphate decarboxylase activity in yeast: 5-fluoro-orotic acid resistance. Mol. Gen. Genet. 197, 345-346.

Brachat, S., Dietrich, F.S., Voegeli, S., Zhang, Z., Stuart, L., Lerch, A., Gates, K., Gaffney, T., Philippsen, P., 2003. Reinvestigation of the Saccharomyces cerevisiae genome annotation by comparison to the genome of a related fungus: Ashbya gossypii. Genome Biol. 4, R45.

Dietrich, F.S., Voegeli, S., Brachat, S., Lerch, A., Gates, K., Steiner, S., Mohr, C., Pohlmann, R., Luedi, P., Choi, S., Wing, R.A., Flavier, A., Gaffney, T.D., Philippsen, P., 2004. The Ashbya gossypii genome as a tool for mapping the ancient Saccharomyces cerevisiae genome. Science 304, 304-307.

Dünkler, A., Wendland, J., 2007. Use of MET3 promoters for regulated gene expression in Ashbya gossypii. Curr. Genet. 52, 1-10.

Fickers, P., Le Dall, M.T., Gaillardin, C., Thonart, P., Nicaud, J.M., 2003. New disruption cassettes for rapid gene disruption and marker rescue in the yeast Yarrowia lipolytica. J. Microbiol. Meth. 55, 727-737.

Fischer, C.R., 1969. Enzymology of the pigmented adenine-requiring mutants of Saccharomyces and Schizosaccharomyces. Biochem. Biophys. Res. Commun. 34, 306-310.

Florea, S., Andreeva, K., Machado, C., Mirabito, P.M., Schardl, C.L., 2009. Elimination of marker genes from transformed filamentous fungi by unselected transient transfection with a Cre-expressing plasmid. Fungal Genet. Biol. 46, 721-730.

Forment, J.V., Ramón, D., MacCabe, A.P., 2006. Consecutive gene deletions in Aspergillus nidulans: application of the Cre/loxP system. Curr. Genet. 50, $217-224$.

Gatignol, A., Baron, M., Tiraby, G., 1987. Phleomycin resistance encoded by the ble gene from transposon Tn5 as a dominant selectable marker in Saccharomyces cerevisiae. Mol. Gen. Genet. 207, 342-348.

Gattiker, A., Rischatsch, R., Demougin, P., Voegeli, S., Dietrich, F.S., Philippsen, P., Primig, M., 2007. Ashbya genome database 3.0: a cross-species genome and transcriptome browser for yeast biologists. BMC Genom. 8, 9.

Gueldener, U., Heinisch, J., Koehler, G.J., Voss, D., Hegemann, J.H., 2002. A second set of loxp marker cassettes for Cre-mediated multiple gene knockouts in budding yeast. Nucleic Acids Res. 30, e23.

Güldener, U., Heck, S., Fiedler, T., Beinhauer, J.D., Hegemann, J.H., 1996. A new efficient gene disruption cassette for repeated use in budding yeast. Nucleic Acids Res. 24, 2519-2524.

Hittinger, C.T., Rokas, A., Carrol, S.B., 2004. Parallel inactivation of multiple GAL pathway genes and ecological diversification in yeasts. Proc. Natl. Acad. Sci. USA $101,14144-14149$.

Iwaki, T., Takegawa, K., 2004. A set of loxP marker cassettes for Cre-mediated multiple gene disruption in Schizosaccharomyces pombe. Biosci. Biotechnol. Biochem. 68, 545-550.

Jiménez, A., Santos, M.A., Pompejus, M., Revuelta, J.L., 2005. Metabolic engineering of the purine pathway for riboflavin production in Ashbya gossypii. Appl. Environ. Microbiol. 71, 5743-5751.

Jiménez, A., Santos, M.A., Revuelta, J.L., 2008. Phosphoribosyl pyrophosphate synthetase activity affects growth and riboflavin production in Ashbya gossypii. BMC Biotechnol. 8, 67-78.
Kaufmann, A., 2009. A plasmid collection for PCR-based gene targeting in the filamentous ascomycete Ashbya gossypii. Fungal Genet. Biol. 46, 595-603.

Kurtzman, C.P., 1995. Relationships among the genera Ashbya, Eremothecium, Holleya, and Nematospora determined from rDNA sequence divergence. J. Ind. Microbiol. 14, 523-530.

Lee, L.M., DaSilva, N.A., 2005. Evaluation of the Saccharomyces cerevisiae ADH2 promoter for protein synthesis. Yeast 22, 431-440.

Magalhães, F., Aguiar, T.Q., Oliveira, C., Domingues, L., 2014. High-level expression of Aspergillus niger - galactosidase in Ashbya gossypii. Biotechnol. Prog. 30 261-268.

Orban, P.C., Chui, D., Marth, J.D., 1992. Tissue- and site-specific DNA recombination in transgenic mice. Proc. Natl. Acad. Sci. USA 9, 6861-6865.

Patel, R.D., Lodge, J.K., Baker, L.G., 2010. Going green in Cryptococcus neoformans: the recycling of a selectable drug marker. Fungal Genet. Biol. 47, 191-198.

Pompejus, M., Revuelta, J.L., Santos, M.A., 1999. Orotidine-5'-Phosphate Decarboxylase-Gene, Gene Construct containing Said Gene and the Utilization Thereof. Patent WO/1999/036432.

Qian, W., Song, H., Liu, Y., Zhang, C., Niu, Z., Wang, H., Qiu, B., 2009. Improved gene disruption method and Cre-loxP mutant system for multiple gene disruptions in Hansenula polymorpha. J. Microbiol. Meth. 79, 253-259.

Ribeiro, O., Gombert, A.K., Teixeira, J.A., Domingues, L., 2007. Application of the Cre-loxP system for multiple gene disruption in the yeast Kluyveromyces marxianus. J. Biotechnol. 131, 20-26.

Ribeiro, O., Wiebe, M., Ilmén, M., Domingues, L., Penttilä, M., 2010. Expression of Trichoderma reesei cellulases CBHI and EGI in Ashbya gossypii. Appl. Microbiol Biotechnol. 87, 1437-1446.

Ribeiro, O., Magalhães, F., Aguiar, T.Q., Wiebe, M.G., Penttilä, M., Domingues, L., 2013. Random and direct mutagenesis to enhance protein secretion in Ashbya gossypii. Bioengineered 4, 322-331.

Sambrook, J., Russell, D.W., 2001. Molecular Cloning: A Laboratory Manual, third ed. Cold Spring Harbor Laboratory Press, New York.

Sauer, B., 1987. Functional expression of the Cre-lox site-specific recombination system in the yeast Saccharomyces cerevisiae. Mol. Cell. Biol. 7, 2087-2096.

Sauer, B., Henderson, N., 1988. Site-specific DNA recombination in mammalian cells by the Cre recombination of bacteriophage P1. Proc. Natl. Acad. Sci. USA 85 5166-5170.

Schlüpen, C., 2003. Untersuchungen zur bedeutung verschiedener enzyme des glycin-stoffwechsels für die riboflavin-bildung in Ashbya gossypii. PhD Thesis. Heinrich Heine University of Düsseldorf, Germany.

Schmitz, H.P., Philippsen, P., 2011. Evolution of multinucleated Ashbya gossypii hyphae from a budding yeast-like ancestor. Fungal Biol. 115, 557-568.

Sherman, F., Fink, G.R., Hicks, J.B., 1986. Methods in Yeast Genetics: A Laboratory Course Manual. Cold Spring Harbor Laboratory Press, New York.

Stahmann, K.P., Revuelta, J.L., Suelberger, H., 2000. Three biotechnical process using Ashbya gossypii, Candida famata, or Bacillus subtilis compete with chemical riboflavin production. Appl. Microbiol. Biotechnol. 53, 509-516.

Steensma, H.Y., Ter Linde, J.J., 2001. Plasmids with the Cre-recombinase and the dominant nat marker, suitable for use in prototrophic strains of Saccharomyces cerevisiae and Kluyveromyces lactis. Yeast 18, 469-472.

Steiger, M.G., Vitikainen, M., Uskonen, P., Brunner, K., Adam, G., Pakula, T., Penttilä, M., Saloheimo, M., Mach, R.L., Mach-Aigner, A.R., 2011. Transformation system for Hypocrea jecorina (Trichoderma reesei) that favors homologous integration and employs reusable bidirectionally selectable markers. Appl. Environ Microbiol. 77, 114-121.

Steiner, S., Wendland, J., Wright, M.C., Philippsen, P., 1995. Homologous recombination as the main mechanism for DNA integration and cause of rearrangements in the filamentous ascomycete Ashbya gossypii. Genetics 140, 973-987.

Sternberg, N., Hamilton, D., 1981. Bacteriophage P1 site-specific recombination: I. Recombination between loxP sites. J. Mol. Biol. 150, 467-486.

Wendland, J., Walther, A., 2005. Ashbya gossypii: a model for fungal developmental biology. Nat. Rev. Microbiol. 3, 421-429.

Wendland, J., Ayad-Durieux, Y., Knechtle, P., Rebischung, C., Philippsen, P., 2000. PCR-based gene targeting in the filamentous fungus Ashbya gossypii. Gene 242, 381-391.

Wendland, J., Dünkler, A., Walther, A., 2011. Characterization of $\alpha$-factor pheromone and pheromone receptor genes of Ashbya gossypii. FEMS Yeast Res. 11, 418-429.

Wright, M.C., Philippsen, P., 1991. Replicative transformation of the filamentous fungus Ashbya gossypii with plasmids containing Saccharomyces cerevisiae ARS elements. Gene 109, 99-105.

Zhang, Y., Liu, H., Li, B., Zhang, J.T., Li, Y., Zhang, H., 2009. Generation of selectable marker-free transgenic tomato resistant to drought, cold and oxidative stress using the Cre/loxP DNA excision system. Transgenic Res. 18, 607-619. 